\title{
Intracranial cell plasma granuloma
}

\author{
Cecília Castelo Branco Brito', Fernanda Cristina Rueda Lopes', \\ Leila Chimelli², Emerson L. Gasparetto ${ }^{3}$
}

Plasma cell granuloma (PCG) are uncommon non-neoplastic masses of unknown etiology, characterized histologically by proliferation of connective tissue with an inflammatory infiltrate of mononuclear elements $^{1,2}$. They occur more commonly in the lung and conducting airways, but can also be seen in other tissues, such as the meninges ${ }^{3}$. The imaging appearance of meningeal PCG can simulate more common neoplasms, as menigiomas. At computed tomography (CT) scans, the meningeal PCG presents as an extra-axial hyperdense mass with homogeneous contrast enhancement. On the magnetic resonance images (MRI), the tumor is characterized by isointense to hypointense signal on T1 weighted-images (WI) and hypointense signal on T2WI, with intense and homogeneous contrast enhancement ${ }^{1,2,4}$.

We report an additional case of meningeal plasma cell granuloma, with emphasis to the differential diagnosis.

\section{CASE}

A 47-year-old man presented with a 1-year history of reduced visual acuity, mainly on the left side, hearing loss, difficulty in walking and urinary retention. The physical examination revealed spastic tetraparesis, ataxia and paresis of VII to XII cranial nerves, beyond left amaurosis, right amblyopia and bilateral hearing loss, worst at right. CT showed a slightly hyperdense and lobulated dural-based mass with intense and homogeneous contrast-enhancement. It was located at the right cerebellum-pontine angle, occupying the basal cisterns, tentorium cerebelli and supra and parasellar regions, compressing the cerebellum, brain stem and aqueduct of Sylvius, resulting in non-communicat- ing hydrocephalus. No bone changes (hyperostosis, permeative and/or destructive lesions) were noticed in the skull base.

The MRI corroborated the relation of the lesion with the duramater, and showed a mass with well-defined lobulated margins, iso to low signal intensity on T1weighted images, low signal intensity on T2-weighted images and homogeneous enhancement after intravenous gadolinium administration (Fig 1 and Fig 2). It occupied the right cerebellum-pontine angle, compressing the brain stem, with probable invasion of both cavernous sinuses. There were multiple other focal masses along the skull base, with partial encasement of vertebral and basilar arteries. The lesions extended to the optic, internal auditory and hypoglossal canals bilaterally. There was a lesion anterior to the medulla oblonga, compressing this structure, with an inferior extension to the anterior aspect of spinal canal.

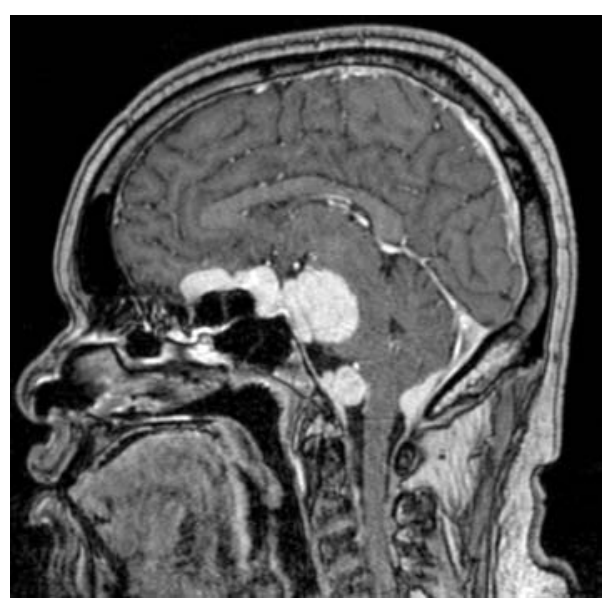

Fig 1. Contrast-enhanced T1-weighted right parasagittal MRI shows the lesions arising from dura at the level of basal cisterns, parasellar region and over the convexity in occipital region. Note the dural tail in the lesions close to the forame magnum.

\section{GRANULOMA PLASMOCITÁRIO INTRACRANIANO}

Received 7 April 2009 Received in final form 8 July 2009 Accepted 12 September 2009

Departments of Radiology and Pathology of the Federal University of Rio de Janeiro, Rio de Janeiro RJ, Brazil: ${ }^{1}$ Mestranda do Programa de Pós-Graduação em Medicina; ${ }^{2}$ Professora Titular de Patologia; 3 Professor Adjunto de Radiologia. 


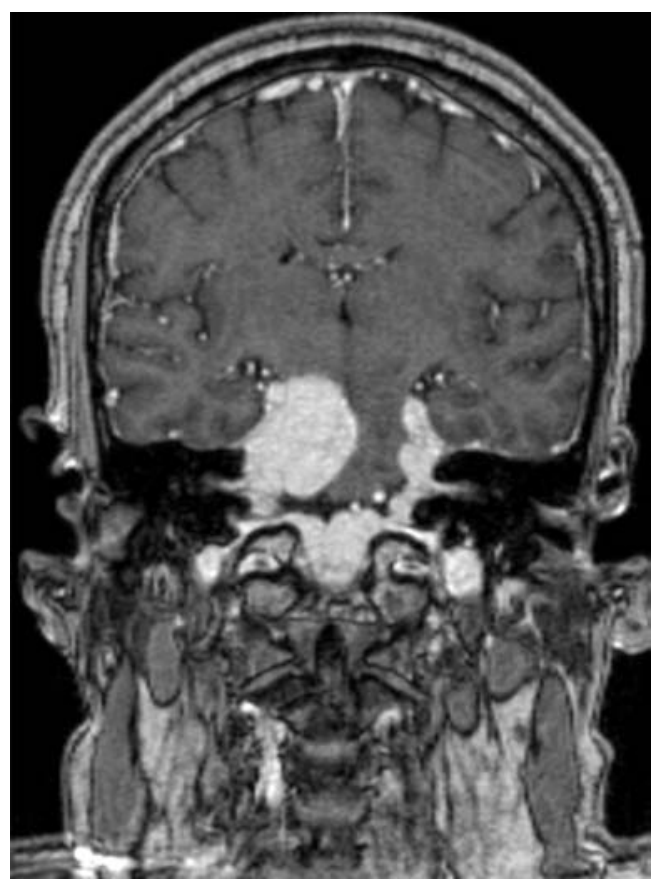

Fig 2. Contrast-enhanced T 1- weighted coronal MRI shows a strong enhanced multifocal lesion with the larger portion located at right cerebellopontine angle cistern, displacing the adjacent brain stem. Notice that the lesions extend to internal auditory and hypoglossal canals bilaterally.

The patient was submitted to a surgical biopsy of the duramater and ventriculo-peritoneal shunt. The histological study showed extensive areas infiltrated predominantly by plasma cells occasionally forming Russell's corpuscles and some lymphocytes (Fig 3). Sections stained for infectious agents where all negative. The final histological diagnosis was plasma cell granuloma. Despite treatment with corticosteroid and radiotherapy, the patient referred progressive worsening of symptoms and progressive gagging for solids and liquids.

\section{DISCUSSION}

Meningeal PCG is a rare intracranial disease with few cases reported ${ }^{1,2}$ and has been referred to by several names in the literature ${ }^{2-6}$, including inflammatory myofibroblastic tumor, fibrohistiocytoma, xanthomatous pseudotumor ${ }^{2}$, fibroxanthoma, xanthogranuloma ${ }^{5}$ and fibroxanthomatous histiocytoma ${ }^{4}$. It occurs in young males (median age, 28 years) and is usually located in the supraand parasellar regions ${ }^{1-3}$, even infiltrating the cavernous sinus $^{2}$. Contiguous paranasal sinus and intracranial disease has been reported ${ }^{3}$. It is a benign tumor with chronic fibrosis and inflammation of the dura and the arachnoid, sometimes affecting the underlying brain ${ }^{3-6}$. The inflammatory infiltrate includes mature plasma cells, polyclonal non-neoplastic $\mathrm{T}$ and $\mathrm{B}$ lymphocytes admixed with macrophages and mononuclear histiocytes. Some meningothelial cells may be found and are considered to be a reactive hyperplasia or a remanescent $t^{6}$. The histopathological findings exclude the major differential diagnosis, such as meningioma and plasmocytoma ${ }^{1,6}$.

The etiology of this lesion is not known, but cases have been described in association with Sjogren's syndrome, sclerosing mediastinitis ${ }^{3}$, polymyositis, neuro-Behçet's disease, Epstein-Barr virus and human immunodeficiency virus infection ${ }^{2}$. It was already proposed that inflammatory pseudotumor is the result of an exaggerated immunological process, which is supported by the evidence of serum immunoglobulin levels elevation. The immunological response may be triggered by a viral infection or may result from an autoimmune disorder ${ }^{2}$. Intracranial lesions have been reported concomitantly with pulmonary lesions, and even occurring after its resection ${ }^{3}$.

The CT scan usually shows a slightly hyperdense, extra-axial mass with homogeneously enhance after contrast administration. The underlying brain can be compressed, with consequent edema. The MRI of meningeal plasma cell granuloma demonstrates a lobulated mass
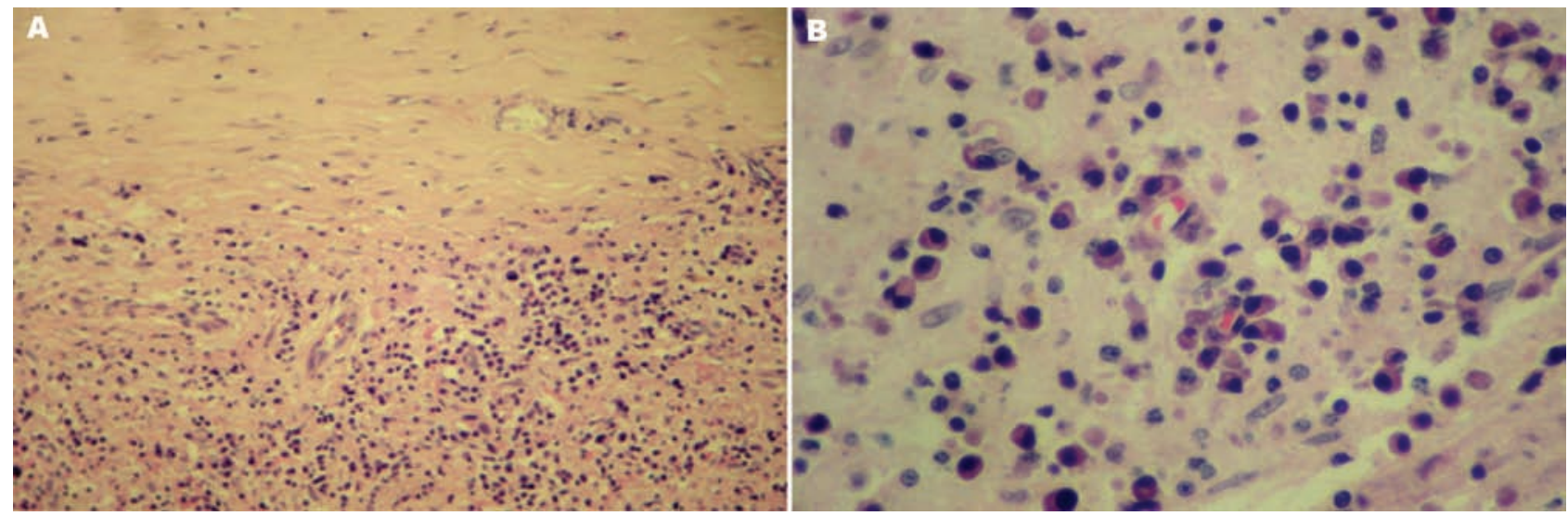

Fig 3. $[A] \times 100$. $[B] \times 400$. HE staining of the duramater showing dense inflammatory mononuclear infiltrate, mainly composed by plasma cells, sometimes volumous and binucleated best seen in B. 
iso to hypointense on T1WI and hypointensity on T2WI, with heterogeneous contrast enhancement ${ }^{2-4}$. The low intensity on T2WI may be due to its high fibrosis content and celularity ${ }^{6}$. It can also have marked hypointensity on susceptible-weighted sequences due to calcification or blood products remanescents ${ }^{7}$. Contrast-enhanced T1WI reveals interdigitation with the adjacent cortex, confirming that these lesions may be locally infiltrative ${ }^{3}$. The tumor is usually located in the parasellar region, with several cases involving the cerebellum-pontine angles ${ }^{2,4,5}$. It is also described as a diffuse dural thickening, even extending to the cervical canal ${ }^{2,5}$. The involvement of base structures such as the Meckel's cavum, cavernous sinus and internal auditory canal can cause cranial nerve palsy ${ }^{7}$.

In our case the lesion was dural based and showed iso to slightly high intensity on T1W) and low intensity on T2WI, with homogenously gadolinium enhancement, corroborating the previous reported cases. The lesion was predominantly located in the right cerebellumpontine angle, with many other foci along the dural base. This topography is not the usual, but there are others similar reports.

The imaging differential diagnosis with meningioma is extremely difficult. The MRI signal and enhancement characteristics are very similar. It can be taken into account epidemiological features, such as sex and age of the patient and the paraselar location to differentiate both lesions $^{1}$. Solitary craniocerebral plasmacytoma also has imaging features similar to PCG. Some differences could be highlighted as lytic lesions of the skull at CT scan and variable signal intensity on T2WI reported in dural plasmacytoma cases. The definitive diagnosis is made by immunohistochemical analysis, which shows a proliferation of polyclonal plasma cells in PCG, unlike the monoclonal population seen in plasmacytoma ${ }^{8}$. Others differential diagnosis of skull base lesions should be done. The hypointense signal on T2WI, is used to differentiate from chordomas, chondrosarcomas, nasopharyngeal carcinomas and metastatic malignant tumors, as these tumors have high signal intensity on T2WI2 .

Steroid therapy is an option for treatment, especially in those patients with involvement of the cavernous sinus ${ }^{2}$. Surgical excision is another choice of treatment ${ }^{4}$, but it may not be curative if the lesion is locally aggressive, sometimes invading bone, and thus nonresectable ${ }^{7}$. Radiation therapy may be an option after incomplete surgical excision ${ }^{7}$. The treatment depends on the location of the tumor. The follow-up is done with MR imaging, evaluating treatment response and tumor recurrence ${ }^{2,5}$. Our patient was submitted to a biopsy to confirm the diagnosis. As the lesion was really advanced by the time of the diagnosis, surgical resection was not possible. Steroids therapy, followed by local radiation, was the treatment option, but the disease progression could not be controlled and the patient is in follow-up.

In summary, meningeal plasma cell granuloma, although rare, should be included in the differential diagnosis of extra-axial enhancing masses, such as meningioma. These lesions share the same imaging findings on both CT scan and MRI. Epidemiological factors and the location of the lesion can help, but the final diagnosis is only defined at the histological examination.

\section{REFERENCES}

1. Murakami M, Hashimoto N, Kimura S, Hosokawa Y, Kakita K. Intracranial plasma cell granuloma with genetic analysis. Acta Neurochir 2003;145:221-225.

2. McCall T, Fassett DR, Lyons G, Couldwell WT. Inflammatory pseudotumor of the cavernous sinus and skull base. Neurosurg Rev 2006;29:194-200.

3. Breidahl WH, Robbins PD, Ives FJ, Wong G. Intracranial plasma cell granuloma. Neuroradiology 2006;38(Suppl):S86-S89.

4. Dwarakanath S, Jaiswal Ak, Ralte AM, Sharma MC, Mahapatra AK. Primary plama cell granuloma of petrous bone. J Clin Neuroscience 2004;11:552-555.

5. Brandsma D, Jansen G, Spliet W, van Nieken K, Taphoorn M. The diagnostic difficulties of meningeal and intracerebral plasma cell granulomas: presentation of three cases. J Neurol 2003;250:1302-1306.

6. Tekkök IH, Ventureyra ECG, Jimenez CL. Intracranial plasma cell granuloma. Brain Tumor Pathol 2000;17:97-103.

7. Makino K, Murakami M, Kitano I, Ushio Y. Primary intracranial plasma-cell granuloma: a case report and review of the literature. Surg Neurol 1995;43:374-378.

8. Provenzale JM, Schaefer P, Traweek ST, et al. Craniocerebral plasmacytoma: MR features. Am J Neuroradiol 1997;18:389-392. 